\title{
Ações de enfermagem prescritas para pacientes internados em um centro de terapia intensiva
}

Nursing actions prescribed to patients in an intensive care unit

Acciones de enfermería prescriptas a pacientes internados en un centro de terapia intensiva

Fernanda Gomes Horta ${ }^{1}$, Patrícia de Oliveira Salgado², Tânia Couto Machado Chianca ${ }^{3}$, Helisamara Mota Guedes ${ }^{4}$

${ }^{1}$ Enfermeira. Diamantina, MG, Brasil. E-mail: fernanda-horta@hotmail.com.

Enfermeira, Doutora em Enfermagem. Professora Assistente da Universidade Federal de Viçosa. Viçosa, MG, Brasil. E-mail: patriciaoliveirasalgado@gmail.com.

${ }^{3}$ Enfermeira, Doutora em Enfermagem. Professora Titular da Escola de Enfermagem da Universidade Federal de Minas Gerais. Belo Horizonte, MG, Brasil. Email: taniachianca@gmail.com.

${ }^{4}$ Enfermeira, Doutora em Enfermagem. Professora Assistente da Universidade Federal dos Vales do Jequitinhonha e Mucuri. Diamantina, MG, Brasil. E-mail: helisamaraguedes@gmail.com.

\section{RESUMO}

Estudo descritivo e documental que objetivou identificar as prescrições de enfermagem elaboradas para pacientes internados em um Centro de Terapia Intensiva. Os dados foram coletados em 231 prontuários, utilizados para documentar as etapas do Processo de Enfermagem. Na amostra de 231 prontuários, identificaram-se 6.197 ações de enfermagem, sendo 3.010 na admissão e 3.187 na alta. Após exclusão das repetições, foram encontradas 104 diferentes ações de enfermagem. Apenas $32(30,76 \%)$ ações de enfermagem foram prescritas para mais de $10 \%$ dos pacientes. Destas, $23(71,87 \%)$ referem-se à rotina hospitalar da unidade e $21(65,62 \%)$ foram prescritas, com maior frequência, no momento da alta em relação ao da admissão. A identificação das intervenções de enfermagem pode auxiliar na construção de um corpo de conhecimentos, na elaboração de protocolos, na fundamentação do ensino e raciocínio clínico, no gerenciamento de custos e no planejamento de alocação de recursos para a qualificação dos serviços de enfermagem.

Descritores: Enfermagem; Processos de Enfermagem; Cuidados de Enfermagem; Unidades de Terapia Intensiva.

\section{ABSTRACT}

A descriptive and documentary study with the aim to identify nursing prescriptions given to patients hospitalized in an Intensive Care Unit. Data were collected from 231 patient charts used to document the Nursing Process steps. Out of this sample of 231 patient charts, we identified 6,197 nursing actions, of which 3,010 were upon admission and 3,187 upon discharge. After excluding repetitions, we found 104 different nursing actions. Only 32 (30.76\%) of which were prescribed for more than $10 \%$ of patients. Of these, $23(71.87 \%)$ referred to the unit's hospital routine and 21 $(65.62 \%)$ were prescribed with greater frequency upon discharge with respect to admission. Identifying nursing interventions can help construct a body of knowledge, create protocols and plan resource allocation for the qualification of nursing services.

Descriptors: Nursing; Nursing Process; Nursing Care; Intensive Care Units.

\section{RESUMEN}

Estudio descriptivo, documental, objetivando identificar las prescripciones de enfermería elaboradas para pacientes internados en un Centro de Terapia Intensiva. Datos recolectados de 231 historias clínicas utilizadas para documentar las etapas del Proceso de Enfermería. En tal muestra, se identificaron 6.197 acciones de enfermería, correspondiendo 3.010 a la admisión y 3.187 al alta. Excluidas las repeticiones, se hallaron 104 diferentes acciones de enfermería. Apenas $32(30,76 \%)$ acciones de enfermería fueron prescriptas para más del $10 \%$ de los pacientes. De ellas, 23 (71.87\%) son rutina hospitalaria de la unidad, y 21 (65,62\%) fueron prescriptas más frecuentemente al momento del alta respecto del momento de admisión. La identificación de las intervenciones de enfermería puede contribuir a construir un cuerpo de conocimientos, en elaboración de protocolos, fundamentación de la enseñanza y razonamiento clínico, gerenciamiento de costos y planificación de localización de recursos para la calificación de los servicios de enfermería.

Descriptores: Enfermería; Procesos de Enfermería; Atención de Enfermería; Unidades de Cuidados Intensivos. 


\section{INTRODUÇÃO}

A Sistematização da Assistência de Enfermagem (SAE) é um conceito amplo utilizado por enfermeiros para organizar e direcionar a assistência fornecida aos pacientes. Neste sentido a SAE possibilita a organização do trabalho profissional quanto ao método, pessoal e instrumentos $^{(1-2)}$.

A importância de se planejar a prática da enfermagem é afirmada pelo Conselho Federal de Enfermagem (COFEN) através da Resolução 358/2009, que recomenda que a SAE deve ser implementada pela operacionalização do Processo de Enfermagem $(P E)^{(1)}$. Este último tem sido visto como um instrumento metodológico que orienta o cuidado profissional de Enfermagem e a documentação da prática profissional.

Em Centros de Terapia Intensiva (CTI) o comprometimento da saúde dos pacientes demanda rapidez de ação da equipe de enfermagem e um cuidado altamente especializado e complexo. Devido a essas características a SAE é instrumento importante na qualidade do atendimento ao paciente crítico.

A enfermagem tem se aproximado dos sistemas de classificação com o objetivo de se afastar do referencial que Ihe guiou nas últimas décadas: o das técnicas e do tratamento curativo e descontextualizado. Busca-se um referencial centrado no cuidado, específico e baseado em evidências $^{(3)}$. Assim, no Brasil, estudos sobre SAE em CTI vêm sendo realizados com o objetivo de analisar a experiência de implantação ${ }^{(4)}$, criar instrumento de coleta de dados $^{(5)}$, construir sistema informatizado(6), levantar diagnósticos e ações de enfermagem ${ }^{(7-8)}$.

Apesar da SAE estar incorporada ao processo de trabalho em algumas instituições de saúde, ainda é necessário o seu aprimoramento, principalmente no que se refere às etapas do PE. Na prática nem todas as etapas são sistematicamente aplicadas. As publicações sobre cuidados de enfermagem são poucas, o que demonstra a necessidade de pesquisas nesse campo de atuação da enfermagem ${ }^{(3,9)}$. Estudos têm revelado dificuldades no estabelecimento e na utilização do processo de enfermagem nas instituições durante os últimos anos, no Brasil e em outros países ${ }^{(10-12)}$.

Pesquisa conduzida em uma unidade semi-intensiva para avaliar a qualidade das anotações de enfermagem mostrou que o percentual de preenchimento completo das intervenções de enfermagem foi de 39,3\%, não sendo estas consideradas de boa qualidade uma vez que o valor esperado era de $80 \%$. O estudo conclui que a baixa qualidade dos registros afeta diretamente 0 cuidado ao paciente, e sugere a capacitação dos profissionais para a execução da SAE, trabalhando com instrumentos aplicáveis a cada realidade para oferecer um cuidado integral e qualificado ao paciente ${ }^{(12)}$.

Um estudo conduzido nos Estados Unidos avaliou se os enfermeiros de um hospital têm documentado no sistema de registro eletrônico a assistência aos pacientes aplicando as etapas do PE e utilizando terminologia padronizada nas diferentes etapas. Os resultados mostram que os profissionais precisam de mais formação para documentar a assistência ao paciente de acordo com o processo de enfermagem e no uso de terminologias, a fim de aumentar a segurança do paciente e melhorar a documentação. de enfermagem ${ }^{(11)}$.

Em um CTI de uma cidade do interior de Minas Gerais, as etapas do PE vêm sendo implementadas desde 2009. É utilizado como arcabouço teórico a teoria das Necessidades Humanas Básicas (NHB) de Wanda de Aguiar Horta ${ }^{(13)}$. A coleta de dados é feita em um instrumento padrão que consta de anamnese e exame físico. O instrumento para registro dos Diagnósticos de Enfermagem (DE) contém 67 diagnósticos de acordo com a taxonomia II da NANDA-International (NANDA$I)^{(14)}$. Estes foram estabelecidos a partir de educação permanente com os enfermeiros da unidade que identificaram os diagnósticos mais frequentes de acordo com a prática vivenciada. O CTI apresenta um roteiro com as principais ações por diagnóstico de enfermagem. Desta forma, o enfermeiro constrói as prescrições de enfermagem de acordo com as necessidades do paciente. A avaliação do paciente é feita diariamente, assim como as outras etapas do processo de enfermagem.

A aplicação efetiva do processo de enfermagem conduz à melhoria da qualidade da assistência de enfermagem e estimula a construção de conhecimentos teóricos e científicos com base na melhor prática clínica ${ }^{(10)}$. Assim, este estudo torna-se relevante à medida que fornece dados teórico-práticos sobre a importância da aplicabilidade do $\mathrm{PE}$, contribuindo para reflexões futuras voltadas à melhoria da assistência de enfermagem em Centros de Terapia Intensiva. Além disso, um conjunto de ações de enfermagem prestadas à pacientes internados em terapia intensiva pode contribuir para a criação de uma base de dados em enfermagem, facilitando a elaboração de protocolos, gerenciamento de custos, dimensionamento de profissionais, tomada de decisão e avaliação da qualidade dos serviços.

Este estudo teve o objetivo de identificar as ações de enfermagem prescritas no momento da admissão e da 
alta para pacientes internados em um Centro de Terapia Intensiva.

\section{METODOLOGIA}

Trata-se de um estudo descritivo e documental, realizado em um hospital geral de referência da cidade de Diamantina, macro e microrregional em assistência médico-hospitalar em média e alta complexidade, localizada no interior de Minas Gerais. Este hospital é o único da cidade que oferece serviços de Terapia Intensiva Adulto.

A população do estudo foi composta pelos prontuários dos pacientes que estiveram internados no CTI no período de março de 2010 a fevereiro de 2011, totalizando 549 prontuários. O cálculo amostral ${ }^{(15)}$ foi realizado considerando um nível de confiança de $95 \%$ e erro aceitável de 5\%. O tamanho da amostra foi definido em 231 prontuários de pacientes.

Foi estabelecido um sistema de contagem para determinar o critério de escolha dos prontuários. Sorteou-se o primeiro número e em seguida pulou dois prontuários. A escolha desses números não foi aleatória, ele é o resultado da razão entre o número total da população pelo número de prontuários analisados $(549 / 231=2,37)$. Esse processo foi repetido até completar o número de prontuários estabelecido no cálculo amostral, 231.

Os dados foram coletados nos prontuários e planilhas utilizadas no CTI para documentar as etapas do PE. Utilizou-se como instrumento uma planilha semiestruturada para transcrição das prescrições de enfermagem no momento da admissão a da alta dos pacientes, além de dados sociodemográficos e clínicos como gênero, idade, diagnóstico médico, período da internação.

Os dados foram analisados utilizando-se o programa Statistical Package for the Social Sciences (SPSS), versão 18.0. Foi realizada análise descritiva com distribuição de frequências simples e percentuais.

Este estudo foi aprovado pelo Comitê de Ética em Pesquisa da Universidade Estadual de Montes Claros por meio da análise do Processo no 2355/2011. Foi resguardado o sigilo e anonimato dos pacientes e dos profissionais envolvidos.

\section{RESULTADOS}

Dos 231 pacientes internados, 134 (58\%) eram do sexo masculino e 97 (42\%) do sexo feminino. A idade média entre eles foi de 53,61 anos com desvio padrão de 21,8 , sendo que o mais velho dos pacientes tinha 96 anos e o mais novo 12 anos. Quanto ao tempo de internação, os pacientes ficaram internados em média 7,7 dias, com desvio padrão de 8,3 sendo que o período de internação mais longo foi de 65 dias e o mais curto de um dia.

De acordo com os diagnósticos médicos, os sistemas do corpo afetados pela doença foram: sistema cardiovascular (87-37,7\%), sistema neurológico (64$27,7 \%)$, sistema respiratório (23-10\%), sistema digestório (22-9,5\%), sistema osteomuscular (15-6,5\%), sistema excretor $(11-4,8 \%)$, sistema reprodutor (5$2,2 \%$ ) e sistema endócrino (4-1,7\%).

Foram identificadas 6.197 ações de enfermagem, sendo 3.010 na admissão e 3.187 na alta. Após exclusão das repetições, foram encontradas 104 diferentes ações de enfermagem no momento da admissão e no momento da alta. Dessas, $32(30,76 \%)$ apresentaram frequência maior que $10 \%$, e $10(9,61 \%)$ apresentaram frequência maior que $50 \%$ (Tabela 1 ).

Durante as admissões foram prescritas, em média, 13,0 ações de enfermagem com desvio padrão de 2,1, enquanto no dia da alta a média de ações prescritas por paciente foi de $13,8 \mathrm{com}$ desvio padrão de 2,7 . 
Tabela 1: Distribuição das ações de enfermagem com frequência maior que $10 \%$, formuladas no momento da admissão e da alta nos prontuários de pacientes internados em um Centro de Terapia Intensiva $(n=231)$. Diamantina, MG, Brasil, 2011.

\begin{tabular}{|c|c|c|c|c|}
\hline \multirow{2}{*}{ Ações de Enfermagem Prescritas } & \multicolumn{2}{|c|}{ Admissão } & \multicolumn{2}{|c|}{ Alta } \\
\hline & $\mathbf{n}$ & $\%$ & $\mathbf{n}$ & $\%$ \\
\hline Realizar higiene oral e ocular & 221 & 95,7 & 220 & 95,2 \\
\hline Realizar balanço hídrico e registrar aspecto da diurese & 220 & 95,2 & 219 & 94,8 \\
\hline Manter cabeceira elevada entre $30-45^{\circ}$ & 208 & 90,0 & 205 & 88,7 \\
\hline Banho de leito & 204 & 88,3 & 168 & 72,7 \\
\hline Realizar hidratação cutânea & 197 & 85,3 & 196 & 84,8 \\
\hline Realizar curativo em acesso venoso & 188 & 81,4 & 178 & 77,1 \\
\hline Avaliar tamanho e fotossensibilidade pupilar & 163 & 70,6 & 155 & 67,1 \\
\hline Avaliar nível de consciência (Glasgow) & 157 & 68,0 & 168 & 72,7 \\
\hline Atentar a frequência cardíaca, $\mathrm{FR}, \mathrm{PA}$, temperatura e perfusão periférica & 155 & 67,1 & 168 & 72,7 \\
\hline Comunicar queixas álgicas & 131 & 56,7 & 128 & 55,4 \\
\hline Comunicar alterações hemodinâmicas & 121 & 52,4 & 104 & 45,0 \\
\hline Realizar mudança de decúbito & 111 & 48,1 & 109 & 47,2 \\
\hline Estimular mudança de decúbito & 98 & 42,4 & 101 & 43,7 \\
\hline Observar padrão respiratório & 75 & 32,5 & 72 & 31,2 \\
\hline Proporcionar um ambiente tranqüilo & 60 & 26,0 & 69 & 29,9 \\
\hline Aspirar TOT e VAS & 59 & 25,5 & 63 & 27,3 \\
\hline Realizar ausculta cardíaca e pulmonar & 57 & 24,7 & 73 & 31,6 \\
\hline Manter colchão caixa de ovo e salva-pés & 46 & 19,9 & 62 & 26,8 \\
\hline Manter sondagem vesical de demora pérvia & 36 & 15,6 & 37 & 16,0 \\
\hline Manter temperatura corporal adequada & 31 & 13,4 & 36 & 15,6 \\
\hline Verificar estase gástrica & 33 & 14,3 & 41 & 17,7 \\
\hline Permitir que o paciente converse & 27 & 11,7 & 36 & 15,6 \\
\hline Banho de aspersão & 26 & 11,3 & 62 & 26,8 \\
\hline Manter AVP pérvio & 26 & 11,3 & 35 & 15,2 \\
\hline Manter grades elevadas & 26 & 11,3 & 36 & 15,6 \\
\hline Manter oximetria de pulso & 26 & 11,3 & 34 & 14,7 \\
\hline Avaliar a pele em busca de vermelhidão & 24 & 10,4 & 33 & 14,3 \\
\hline Sinais flogísticos na incisão dos cateteres & 24 & 10,4 & 29 & 12,6 \\
\hline Fornecer informações & 23 & 10,0 & 24 & 10,4 \\
\hline Conter paciente & 21 & 9,1 & 44 & 19,0 \\
\hline Observar sinais de choque & 22 & 9,5 & 24 & 10,4 \\
\hline Manter paciente orientado no tempo e no espaço & 18 & 7,8 & 26 & 11,3 \\
\hline
\end{tabular}

\section{DISCUSSÃO}

A maioria dos pacientes da amostra estudada é do sexo masculino (58\%). Estudos mostram que, em geral, pacientes do sexo masculino apresentam maior risco para as principais causas de morte, assim, observa-se que os homens adoecem mais do que as mulheres ${ }^{(16-18)}$.

A idade média encontrada foi de 53,6 anos, apesar de estudos apontarem que nos últimos anos esta média de idade dos pacientes internados em terapia intensiva tem aumentado em consequência do envelhecimento populacional $^{(17-19)}$.

A média de permanência dos pacientes internados foi de sete dias, maior do que quando comparada ao outro estudo desenvolvido em CTI, onde a média registrada foi de 4,3 dias $^{(16)}$. A média de dias de internação encontrada pode estar relacionada à inexistência de uma unidade destinada a cuidado semiintensivo na instituição. Este fato também é identificado em outro estudo como um dos fatores que pode dificultar a redução no tempo de internação de pacientes em
CTI's ${ }^{(20)}$.

Entre os problemas de saúde identificados, as causas mais frequentes de internação foram as doenças do sistema cardiovascular. Esse resultado corrobora dados da Secretaria de Vigilância em Saúde/Ministério da Saúde que identificou no período compreendido entre 1980 e 2008 que as doenças do sistema circulatório representaram a principal causa de óbitos no Brasil, correspondendo a $32 \%$ do total de óbitos nesses anos ${ }^{(17)}$.

As prescrições de enfermagem que apresentaram frequência maior que $50 \%$ na amostra estudada coincidem com os cuidados encontrados em outros estudos em $\mathrm{CTIs}^{(8-9)}$, e que incluem: realizar higiene oral e ocular; realizar balanço hídrico e registrar aspecto da diurese; manter cabeceira elevada entre 30-450; realizar banho de leito; realizar hidratação cutânea; realizar curativo em acesso venoso; avaliar tamanho e fotossensibilidade pupilar; avaliar nível de consciência (Glasgow), atentar a frequência cardíaca, FR, PA, temperatura e perfusão periférica; e comunicar queixas 
álgicas. Essas ações de enfermagem identificadas também constam como frequentemente prescritas para pacientes críticos em outros estudos ${ }^{(9,21)}$. Percebe-se que os cuidados de enfermagem identificados são importantes à prática clínica em CTI. Tal fato se justifica por se referirem a aspectos de autocuidado, ao controle das eliminações e ao controle de risco, conforme identificadas em outro estudo ${ }^{(9)}$. Em especial assinala-se que este perfil de pacientes apresenta dificuldade para realizar atividades por si próprio, necessitando de intervenções de realização das atividades de vida diária do indivíduo e de promoção de conforto ${ }^{(9)}$.

Entre os cuidados prescritos com frequência maior que $10 \%, 20(62,5 \%)$ foram considerados cuidados que fazem parte da rotina hospitalar, como: "banho de leito", "banho de aspersão", "higiene oral", "fornecer informações". Fica evidente que em CTI, além da prescrição de cuidados intensivos como monitoração hemodinâmica invasiva, controle hídrico, cuidados com ventilação mecânica, entre outros, os enfermeiros, em sua maioria, prescrevem uma grande quantidade de ações rotineiras, que deveriam ser parte dos protocolos da unidade. O mesmo também foi observado em outros estudos $^{(8,22-23)}$. Os cuidados prescritos pelos enfermeiros devem ser revistos de forma a englobar prescrições focadas em cuidados rotineiros básicos. Os enfermeiros devem ser capacitados a elaborar prescrições com descrições de ações que possam colaborar na resolução dos problemas existentes e apropriadas para cada caso clínico ${ }^{(9)}$.

Por sua vez, cerca de $70 \%$ dos cuidados encontrados, obtiveram frequência menor que $10 \%$ nas prescrições por paciente do dia de admissão e da alta, revelando que o maior número de cuidados foram prescritos para um número reduzido de pacientes, sendo que para cada paciente foram selecionadas ações específicas, na tentativa se solucionar o seu problema.

Ao comparar a média de ações de enfermagem prescritas por pacientes na admissão (13) e na alta $(13,8)$ observou-se um aumento de 0,8 na média de cuidados prescritos na alta. Verifica-se, por exemplo, que as ações de enfermagem "avaliar nível de consciência (Glasgow)", "atentar a frequência cardíaca, FR, PA, temperatura e perfusão periférica", "proporcionar um ambiente tranquilo", "aspirar TOT e VAS", entre outras, foram prescritas para um maior número de pacientes no momento da alta do que na admissão. Este fato pode ser explicado porque durante a admissão o paciente é desconhecido para a equipe, além de estar grave, necessitando de cuidados intensivos, portanto, requer mais atenção da equipe o que pode comprometer o tempo dispensado para a documentação dos cuidados. $\mathrm{Na}$ alta o paciente já se apresenta em melhores condições de saúde sendo disponibilizado mais tempo para a documentação.

Durante a alta do paciente, além dos cuidados referentes à recuperação e prevenção de agravos, é de suma importância que no plano de cuidados também sejam incluídas as orientações de alta a serem fornecidas pela equipe de enfermagem ao paciente e família(24), objetivando o preparo dos mesmos para a saída do CTI. Estas orientações caracterizam-se como um cuidado de enfermagem, podendo levar a um maior número de ações de enfermagem prescritas no dia de alta visando contemplar a continuidade do cuidado.

Os resultados obtidos neste estudo possuem o seu maior foco nas intervenções utilizadas na prática clínica de enfermagem intensivista e se somam ao conhecimento produzido em recentes publicações no que diz respeito às etapas do $P E$. Destacando-se o raciocínio clínico que está presente em todas as ações e decisões assistenciais do enfermeiro: no diagnóstico dos problemas, na escolha de intervenções apropriadas e na avaliação dos resultados obtidos, além das etapas de planejamento e implementação das intervenções de enfermagem ${ }^{(9-10,25)}$. Assim, espera-se que esta investigação possa auxiliar a Enfermagem a apropriar-se, cada vez mais, dos fenômenos da sua prática como o diagnosticar e o prescrever ações com o uso de linguagem própria descrita pelos sistemas de classificações existentes.

Torna-se importante valorizar os momentos de educação permanente com a equipe de enfermagem, para que ações sejam implementadas no hospital com o intuito de aperfeiçoar o processo de enfermagem, melhorar a qualidade das anotações de enfermagem, discutir o tempo de permanência de alguns DE e intervenções de enfermagem, elaboração de um plano de alta que facilite a continuidade do cuidado para que o $P E$ seja cada vez mais efetivo e resolutivo.

\section{CONCLUSÃO}

Este estudo permitiu identificar os cuidados de enfermagem prescritos a pacientes internados em um CTI. Foram identificadas 104 diferentes ações de enfermagem prescritas para os pacientes tanto na admissão e na alta. As prescrições de enfermagem que apresentaram frequência maior que $50 \%$ na amostra estudada referem-se à ações/atividades de autocuidado, de controle das eliminações e de controle de risco dos 
pacientes, o que condiz com as necessidades de pacientes críticos. Contudo, também foram identificadas ações de enfermagem relativas aos cuidados rotineiros como "banho de leito", "banho de aspersão", "higiene oral", "fornecer informações". Observou-se que não houve diferenciação entre ações prescritas na admissão e na alta de pacientes críticos. Entretanto, a alta de um CTI trata-se de um momento de grande importância para assegurar a continuidade do cuidado e evitar reinternações, que contribuem significativamente para elevar as despesas na atenção à saúde. É necessário que a alta seja planejada e sistematizada, garantindo um esclarecimento maior, tanto para o paciente quanto para a equipe de enfermagem que irá recebê-lo na unidade de internação. Acredita-se que o plano de cuidados no momento da alta deverá conter informações referentes

\section{REFERÊNCIAS}

1. Conselho Federal de Enfermagem. Resolução n³58, de 15 de outubro de 2009. Dispõe sobre a Sistematização da Assistência de Enfermagem e a implementação do Processo de Enfermagem em ambientes, públicos ou privados, em que ocorre o cuidado profissional de Enfermagem, e dá outras providências. Brasília, 2009. [cited 2014 mai 10]. Available from: http://site.portalcofen.gov.br/node/4384.

2. Carvalho EC, Bachion MM. Processo de enfermagem e sistematização da assistência de enfermagem - intenção de uso por profissionais de enfermagem. Rev. Eletr. Enf. [Internet]. 2009 [acesso em: 14 mai 2014]; 11(3):466. Disponível em http://www.fen.ufg.br/revista/v11/n3/v11n3a01.htm.

3. Truppel TC, Meier MJ, Calixto RC, Peruzzo SA, Crozeta K. Sistematização da Assistência de Enfermagem em Unidade de Terapia Intensiva. Rev bras enferm. 2009; 62(2):221-27. 4. Amante LN, Rossetto AP, Schneider DG. Sistematização da Assistência de Enfermagem em Unidade de Terapia Intensiva sustentada pela Teoria de Wanda Horta. Rev Esc Enferm USP. 2009; 43(1):54-64.

5. Silva RS, Pereira A, Ribeiro AG, Marinho CMS, Carvalho IS, Ribeiro R. Elaboração de um instrumento para coleta de dados de paciente crítico: histórico de enfermagem. Rev. enferm. UERJ. 2012; 20(2):267-73.

6. Barra DCC, Dal Sasso GTM. Tecnologia móvel à beira do leito: processo de enfermagem informatizado em terapia intensiva a partir da cipe $1.0 \AA$. Texto contexto - enferm. [Internet]. 2010 [acesso em: 09 mai 2014]; 19(1): 54-63. Disponível em: http://www.scielo.br/scielo.php?script=sci_arttext\&pid=S010407072010000100006\&lng=en.

7. Salomé GM. Diagnóstico de enfermagem dos pacientes internados em uma Unidade de Terapia Intensiva. Saúde Coletiva. $2011 ; 47(8): 24-8$.

8. Salgado PO, Chianca TCM. Identification and mapping of the nursing diagnoses and actions in an Intensive Care Unit. Rev. Latino-Am. Enfermagem. 2011; 19(4):928-35.

9. Lucena AF, Gutiérrez MGR, Echer IC, Barros ALBL.

Intervenções de enfermagem utilizadas na prática clínica de uma uni $\neg$ dade de terapia intensiva. Rev. Latino-Am. Enfermagem [Internet]. 2010 [acesso em: 10 maio 2014]; 18(5): 873-880. Disponível em:

http://www.scielo.br/scielo.php?script=sci_arttext\&pid=S010411692010000500006\&lng=pt.

10. Pokorski S, Moraes MA, Chiarelli R, Costanzi AP, Rabelo ER. Nursing process: from literature to practice. What are we actually doing? Rev. Latino-Am. Enfermagem. 2009; 17(3):302-7. 11. Häyrinen K, Lammintakanen J, Saranto K. Evaluation of às orientações de alta, o que não ocorreu. Além disso, deve estabelecer uma interação entre os profissionais envolvidos no processo saúde-doença, para superar a fragmentação do cuidado, permitindo a discussão sobre a resolução de problemas, com vistas à compreensão da realidade vivida pelo paciente.

As implicações para a prática clínica de enfermagem referem-se à identificação de um conjunto de ações de enfermagem utilizadas por enfermeiros intensivistas em sua prática cotidiana que podem auxiliar na construção de um corpo de conhecimentos, baseado em evidências de cuidado a pacientes internados em terapia intensiva, na elaboração de protocolos clínicos, na fundamentação do ensino e do raciocínio clínico, no gerenciamento de custos e no planejamento de alocação de recursos para a qualificação dos serviços de enfermagem.

electronic nursing documentation--nursing process model and standardized terminologies as keys to visible and transparent nursing. Int J Med Inform. 2010; 79(8):554-64.

12. Silva JÁ, Grossi ACM. Haddad MCL, Marcon SS. Avaliação da qualidade das anotações de enfermagem em unidade semiintensiva. Esc. Anna Nery. 2012; 16 (3): 576-581.

13. Horta WA. Processo de Enfermagem. São Paulo: Editora Pedagógica e Universitária; 1979.

14. NANDA-International. Diagnósticos de enfermagem da NANDA: definições e classificação 2012-2014. Porto Alegre: Artmed; 2013. 606 p.

15. Barbeta PA, Reis MM, Bornia AC. Estatística: para cursos de engenharia e informática. São Paulo: Atlas; 2010.

16. Paganin A, Menegat P, Klafke T, Lazzarotto A, Fachinelli TS, Chaves IC, et al. Implantação do diagnóstico de enfermagem em unidade de terapia intensiva: uma análise periódica. Rev Gaúcha Enferm [Internet]. 2010 [acesso em: 10 mai 2014];31(2):307-13. Disponível em: http://seer.ufrgs.br/RevistaGauchadeEnfermagem/article/view/1 1428/10240.

17. Ministério da Saúde. Secretaria de Vigilância em Saúde, Departamento de Análise de Situação de Saúde. Saúde Brasil 2009: uma análise da situação de saúde e da agenda nacional e internacional de prioridades em saúde. Brasília: Ministério da Saúde; 2010.

18. Ramos GS, Santana LC, Ferreira PHC, Chianca TCM, Guedes HM. Diagnósticos de enfermagem documentados em prontuários de pacientes em Unidade de Terapia Intensiva. R. Enferm. Cent. O. Min. 2013; 3(2):679-86.

19. Salgado PO, Melo LS, Souza LME, Andrade PGR. Comparison of the workload of nursing staff in adult intensive care unit. J Nurs UFPE on line. [Internet]. 2012 [acesso em: $20 \mathrm{dez}$ 2014];6(4):773-8. Disponível em:

http://www.revista.ufpe.br/revistaenfermagem/index.php/revista /article/viewArticle/2339.

20. Moock M. Casos clínicos em terapia intensiva. Rev bras ter intensiva. 2006; 18(1):12.

21. Wong E, Scott LM, Briseno JR, Crawford CL, Hsu JY. Determining critical incident nursing interventions for the critical care setting. Int J Nurs Terminol Class. 2009; 20(3):110-21 22. Aquino DR, Filho WDL. Construção da Prescrição de Enfermagem Informatizada em uma UTI. Cogitare Enferm. 2004;9(1):60-70.

23. Pivotto F, Filho WDL, Lunardi VL. Prescrição de enfermagem: dos motivos da não realização às possíveis estratégias de implementação. Cogitare enferm. 2004; 9(2):32-42.

24. Guedes HM, Santos WL, Nakatani AYK, Chianca TCM. 
Diagnósticos de enfermagem na admissão e alta hospitalar de idosos. Rev. enferm. UERJ. 2011;19(4):564-70.

25. Cruz DALM, Cerullo JASB. Raciocínio clínico e pensamento crítico. Rev. Latino-Am. Enfermagem. 2010;18(1):124-9.

Artigo recebido em 31/01/2013.

Aprovado para publicação em 06/05/2014.

Artigo publicado em 30/09/2014. 